\title{
Advanced Fusion and Empirical Mode Decomposition-Based Filtering Methods for Breathing Rate Estimation from Seismocardiogram Signals
}

\author{
Christina Kozia *,+(D) and Randa Herzallah ${ }^{+}$(D) \\ Department of Mathematics, School of Engineering and Applied Sciences, Aston University, Aston St., \\ Birmingham B4 7ET, UK; r.herzallah@aston.ac.uk \\ * Correspondence: kozia.christina@gmail.com \\ + These authors contributed equally to this work.
}

Citation: Kozia, C.; Herzallah, R

Advanced Fusion and Empirical

Mode Decomposition-Based Filtering Methods for Breathing Rate Estimation from Seismocardiogram Signals. Information 2021, 12, 368. https://doi.org/10.3390/info12090368

Academic Editor: Khalid Sayood

Received: 4 August 2021

Accepted: 10 September 2021

Published: 11 September 2021

Publisher's Note: MDPI stays neutra with regard to jurisdictional claims in published maps and institutional affiliations.

Copyright: (c) 2021 by the authors. Licensee MDPI, Basel, Switzerland. This article is an open access article distributed under the terms and conditions of the Creative Commons Attribution (CC BY) license (https:// creativecommons.org/licenses/by/ $4.0 /$ )
Abstract: Breathing Rate (BR), an important deterioration indicator, has been widely neglected in hospitals due to the requirement of invasive procedures and the need for skilled nurses to be measured. On the other hand, biomedical signals such as Seismocardiography (SCG), which measures heart vibrations transmitted to the chest-wall, can be used as a non-invasive technique to estimate the BR. This makes SCG signals a highly appealing way for estimating the BR. As such, this work proposes three novel methods for extracting the BR from SCG signals. The first method is based on extracting respiration-dependent features such as the fundamental heart sound components, S1 and S2 from the SCG signal. The second novel method investigates for the first time the use of data driven methods such as the Empirical Mode Decomposition (EMD) method to identify the respiratory component from an SCG signal. Finally, the third advanced method is based on fusing frequency information from the respiration signals that result from the aforementioned proposed methods and other standard methods. The developed methods in this paper are then evaluated on adult recordings from the combined measurement of ECG, the Breathing and Seismocardiograms database. Both fusion and EMD filter-based methods outperformed the individual methods, giving a mean absolute error of 1.5 breaths per minute, using a one-minute window of data.

Keywords: breathing rate estimation; non-invasive monitoring; seismocardiogram; empirical mode decomposition; fusion methods; frequency domain analysis; autoregressive analysis

\section{Introduction}

The Breathing Rate (BR) plays a key role in patient monitoring as it describes the air in and out of lungs, which can be an indicator of deterioration as the body attempts to maintain oxygen delivery to the tissues [1]. A study, investigating early signs of deterioration, has shown that patients in critical status could have been identified as high risk up to $24 \mathrm{~h}$ previously, indicating that lack of vital signs monitoring, such as the BR, can result in poor outcomes for patients [2]. Another study conducted in hospitals in 1993 indicated that a BR greater than 27 breaths per minute $(\mathrm{bpm})$ is the most important indicator that can predict failure of the heart to contract effectively [3]. Moreover, the necessity of BR was investigated in another study, and it was claimed that $21 \%$ of hospitalised patients with a BR of 25-29 bpm assessed by a critical care outreach service die in hospital [4]. An increase of the rate of mortality has been also reported for patients with higher BR [4]. It has been demonstrated that just over half of the unhealthy subjects suffering a serious event on the hospital wards have a BR greater than $24 \mathrm{bpm}$ and these subjects could have been identified as high risk up to $24 \mathrm{~h}$ before the event [4]. Furthermore, it has been reported that $\mathrm{BR}$ is a better patient status indicator because of its relative greater changes compared to heart rate or blood pressure [2]. Therefore, the necessity of monitoring the BR is evidently clear based on the aforementioned studies. However, it has been demonstrated 
that BR is often neglected due to patients' acuity, lack of time, inadequate nursing skills, and cumbersome wearable sensors $[2,5,6]$. Traditionally, the BR is extracted by measuring body volume changes around the thorax over a period of time, which is proven insufficient and time-consuming especially in case of emergency [2]. In addition, most of the electronic methods for BR monitoring require the use of cumbersome devices, such as chest bands for inductance plethysmography, which cause patients to feel discomfort during the recording $[7,8]$.

As a result, several methods have been developed over the past years investigating the extraction of the BR using non-invasive techniques from signals such as the Electrocardiogram (ECG), the Photoplethysmogram (PPG) [9], and the Seismocardiogram (SCG) [10-15] which are widely monitored in hospitals. However, most of these methods rely on extracting and identifying features like the heartbeat complexes, which result in a lower expected accuracy of BR estimation. This means that alternative methods are still necessary to be developed, and the accuracy of the BR estimations from these signals still needs to be enhanced and improved through the development of alternative methods. As mentioned previously, SCG is a non-invasive technique which measures waves produced by the heart acceleration and deceleration due to the heart wall motion and blood movement $[16,17]$. It is commonly recorded by accelerometers placed either on the left clavicle of sternum, which makes its acquisition inexpensive and of high quality [18]. Therefore, this work will investigate the development of alternative methods for extracting the BR from SCG signals.

More specifically, this paper proposes three novel methods for extracting the respiratory signal from SCG recordings. The first method is based on the amplitude variations of the SCG signal due to respiration. As will be shown from further development, the accuracy of these methods relies on the accurate identification of the S1-peaks from the SCG recording. Our second method looks at the development of a novel filter-based method which applies for the first time the EMD on the SCG signal. Empirical Mode Decomposition (EMD) is not new though, it has been widely used in the literature to identify masked signals in the ECG, such as respiration [19]. It also has been used in other biomedical related applications such as the evaluation of voice disorders [20,21]. A review on the use of EMD in biomedical signals and image processing can also be found in [22]. The idea behind this method is to investigate the ECG signal components in the frequency domain in order to choose which component corresponds to the respiration signal. In our proposed method, EMD is used for the first time to decompose the SCG signal into its oscillatory components from which the respiration component is then identified based on a spectral approach. The major advantage of this proposed approach is that it does not require any pre-processing before applying the EMD, compared to the feature-based methods which involve the pre-processing of both the SCG and ECG signals. Our third method looks at the development of an advanced method fusion technique to further improve the accuracy of BR estimation. The proposed method fusion techniques is based on an all-pole Autoregressive (AR) model and a pole magnitude criterion. A critical point of AR modeling is the model order selection. The majority of studies decide the model order experimentally by testing orders ranging from 6 to 20 [23]. However, this approach of order selection is lurking risks as it is referring to a specific age and type of patients, thus it may deteriorate the BR estimation accuracy for subjects with different characteristics. This drawback is addressed in our suggested enhanced fusion method by introducing a model order selection criterion which is based on the partial autocorrelation function (PACF) of the respiration signal.

To achieve the objective of this work, the paper is organised as follows: Section 2 discusses the data that is used for the demonstration of the theoretical development of this paper. The standard methods for extracting the respiratory signal from SCG recordings are discussed in Section 3. Section 4 provides a detailed discussion on the methods proposed in this work for the extraction of respiratory signals from SCG recordings. Time and frequency domain analysis of respiratory signals are discussed in Section 5. Section 6 describes the measures used to assess the developed methods in this paper. The results are given in 
Section 7. Section 8 discusses the proposed fusion method and provides the results of its implementation. Further analysis to what has been achieved in this paper is provided in Section 9. Finally, Section 10 analyses the strengths and limitations of this study and offers perspectives for future work.

\section{Database Used}

The developed methods were tested on SCG recordings from the combined measurement of ECG, Breathing, and Seismocardiograms database (CEBSDB) [24]. The CEBSDB database was selected as it is publicly available on Physionet [25] and contains SCG recordings and reference BR information. When the database was developed, data were acquired by 20 presumed healthy volunteers. During the validation of our algorithms we used all 20 available recordings and we did not excluded any of them.

In more details, the SCG signals belong to healthy male and female adult volunteers, who were asked to be very still in supine position and their ages range from 19 to 30 years old. The duration of the SCG signals is $5 \mathrm{~min}$ and the sampling frequency was set at $5 \mathrm{kHz}$. A reference $B R$ is also provided for each minute which varies from 10 to $26 \mathrm{bpm}$. The code name of the SCG adult recordings is b00, followed by the volunteer number.

\section{SCG-Derived Respiration: Standard Methods}

This section investigates the current state of the art methods which attempt the extraction of respiratory signals from SCG recordings. A study conducted in 2012 [14] lists three respiration-dependent SCG features which arise from the variations in timings and intensity of the primary heart sounds, S1 and S2 of the SCG signal. It was also made clear that R-peak identification plays a major role in SCG analysis, as the R-peak is used as a fiducial point in order to identify critical points in the SCG signal.

\subsection{S1-S1 Interval Derived Respiratory Signal}

The first respiratory-dependent SCG feature is the S1-S1 interval, which is defined as the time interval between two consecutive S1-peaks in the SCG signal. The S1-peak is set to be the maximum amplitude of the SCG signal in a $0.1 \mathrm{sec}$ time interval following the R-peak. Figure 1 shows a small part of the ECG signal which belongs to subject b001. For each R-peak (red asterisks in Plot 1), an S1-peak is identified in the SCG signal, and it is represented by black circles in Plot 2. For the identification of the R-peaks in the ECG signal, we used an adaptive detection algorithm that makes use of EMD of the latter [26-28].

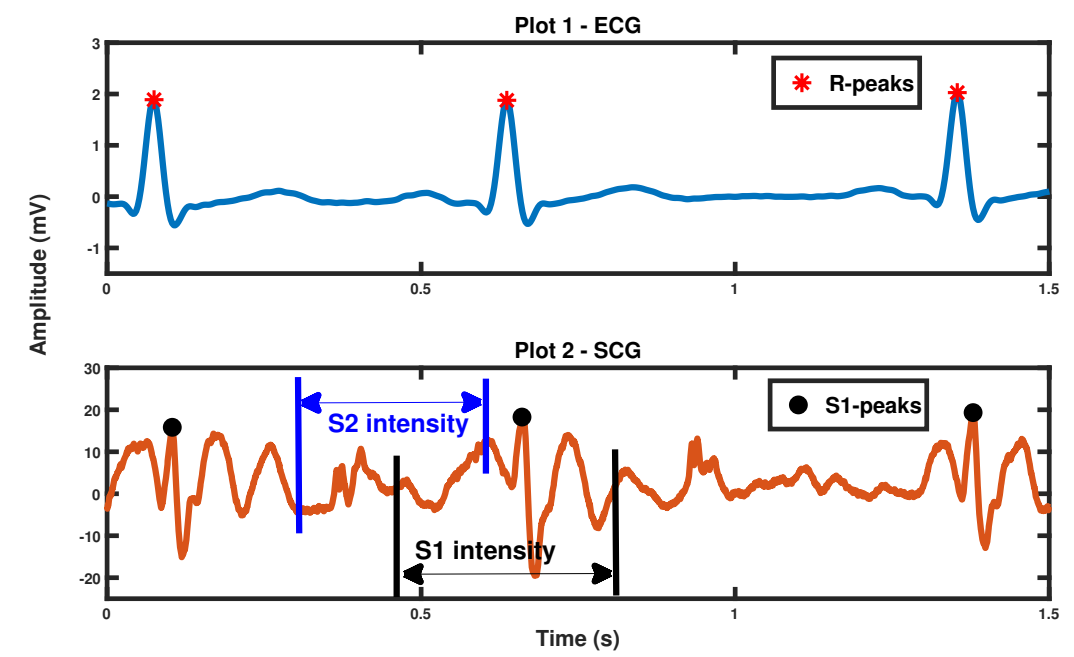

Figure 1. S1-peak identification on SCG: Plot 1 shows the ECG signal of subject b001, along with the detected R-peaks (red asterisks). Plot 2 shows the corresponding SCG signal, along with the identified S1-peaks (black circles). In addition, the time intervals for the S1- and S2-intensity are defined. 


\subsection{S1 Intensity, S2 Intensity Derived Respiratory Signal}

As previously mentioned, additional respiration-dependent features were explored in [14], which are based on the intensity of the S1 and S2 primary sounds of the SCG signal. The S1 intensity is defined as the Root Mean Square (RMS) of the SCG windowed at $0.2 \mathrm{~s}$ before and $0.15 \mathrm{~s}$ after the S1-peak. Figure 1 Plot 2 defines the S1 interval used in the S1 intensity method. Similarly, S2 intensity is calculated as the RMS of the SCG windowed at $0.22 \mathrm{~s}$ to $0.5 \mathrm{~s}$ following the S1-peak. The S2-interval used in the S2 intensity method is also depicted in Figure 1 Plot 2. Moreover, the S1/S2 intensity ratio was calculated as a respiratory-measure that can extract respiration from the SCG signal.

The aforementioned features (S1-S1 interval, S1 and S2 intensity) create discrete non-uniform time series which are sampled at the heart rate (HR) frequency because their location depends on the R-peaks in the ECG signal. Therefore, these time series are down-sampled at $8 \mathrm{~Hz}$ using cubic spline interpolation, in order to get uniformly sampled respiration signals. Furthermore, as will be seen from further developments, in our implementations, the accuracy of the BR estimation is increased by further processing the uniformly sampled respiration signal by a band-pass filter, whose cut-off frequencies are at $0.0666 \mathrm{~Hz}$ and $0.5 \mathrm{~Hz}$. The range of respiration frequencies was determined as follows: The lower limit was fixed at $0.0666 \mathrm{~Hz}(4 \mathrm{bpm})$ [7]. The upper limit was set to $0.5 \mathrm{~Hz}(30 \mathrm{bpm})$ in order to include all respiration frequencies that can be found in the database used.

\section{SCG-Derived Respiratory Signal: Proposed Advanced Methods}

Thus far, current state-of-the-art studies have focused on the extraction of respiratory signals that are based on measuring frequency modulations from features such as the S1-S1 interval and the S1, S2, S1/S2 intensity. What it missing in the current literature is the impact of respiration on the amplitude of the S1-peaks. Therefore, amplitude modulation of the S1-peaks will be investigated for the first time in this work to extract the respiratory signal from SCG recordings. Furthermore, an apparent disadvantage of feature-based respiratory signals extraction methods is that they require the identification of both the Rand S1-peaks, which can introduce delays in the estimation and increase the computational cost. Thus, the need for a filter-based technique which does not demand the detection of the S1-peaks is evident. Consequently, the exploitation of the EMD method for the extraction of respiration signals from SCG recordings will be explored for the first time in this paper.

\subsection{S1 Peak Amplitude Modulation Derived Respiratory Signal}

This method takes advantage of the Amplitude Modulation (AM) of the S1-peaks over time, assuming that these modulations are induced by respiration. The S1-peaks are detected with respect to the locations of the R-peaks, as described previously. Then, the amplitude of the S1-peaks is computed and kept for further processing. The AM feature produces a discrete non-uniform time series which is sampled at the HR frequency. Therefore, the time series are interpolated and then down-sampled at $8 \mathrm{~Hz}$, in order to get a uniformly sampled respiration signal. Finally, the respiration signal is filtered within reasonable respiration frequencies $(0.0666-0.5 \mathrm{~Hz})$.

\subsection{Empirical Mode Decomposition Derived Respiratory Signal}

It has been previously mentioned that the EMD method can possibly extract signals which are hidden in an observed signal, such as the ECG. Therefore, this work researches its potential application to the SCG recordings as a means of extracting the respiration signal. The accelerometers, used in the SCG signal acquisition, are usually located on the subject's chest, thus the respiration signal is expected to be embedded in an SCG signal. Therefore, the EMD method is proposed here as a potential approach to extract the respiration signal from the SCG signal.

In order to extract the respiration signal, first the Intrinsic Mode Functions (IMFs) of the SCG signal are obtained after applying the EMD method. The selection of the respiratory 
IMF is then based on a spectral analysis. The IMF which contains the peak frequency that lies within the respiratory frequency range is set to be the EMD-Derived Respiration (EMDDR) signal. Figures 2-4 show the extracted IMFs after applying the EMD on the SCG signal of subject b001. Moreover, the frequency spectrum after applying a Discrete Fourier Transform (DFT) of each IMF is also depicted in the aforementioned figures.

It can be observed that the frequency content of the lower order IMFs (Figures 2 and 3), which represent the fast oscillations of the signal, is widely spread and there is no clear dominant frequency, thus these IMFs are excluded from our analysis in order to estimate the BR. The last IMFs (Figure 4) represent the slow oscillations that can be found in the SCG signal. Closer inspection of Figure 4 (Plots 1 and 2) reveals that the spectrum of IMF 17 has a dominant frequency which lies between $0.0666 \mathrm{~Hz}$ and $0.5 \mathrm{~Hz}$. Moreover, it can be observed that the dominant frequency of IMF 17 is more distinct, compared to the frequency peaks of IMF 18 (Plot 4).


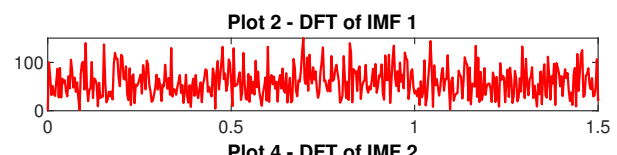

Plot 4 - DFT of IMF 2


Plot 6 - DFT of IMF 3

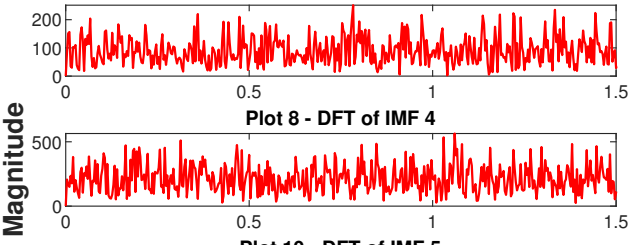

Plot 10 - DFT of IMF 5
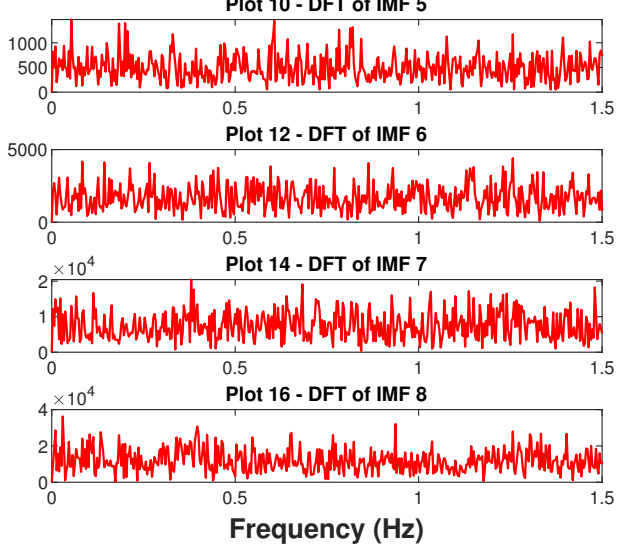

Figure 2. DFT analysis of the IMFs 1 to 8 of b001: The plots on the left column depict IMFs 1 to 8 . The plots on the right column show the corresponding spectra (DFT points $=300,000$ ). 

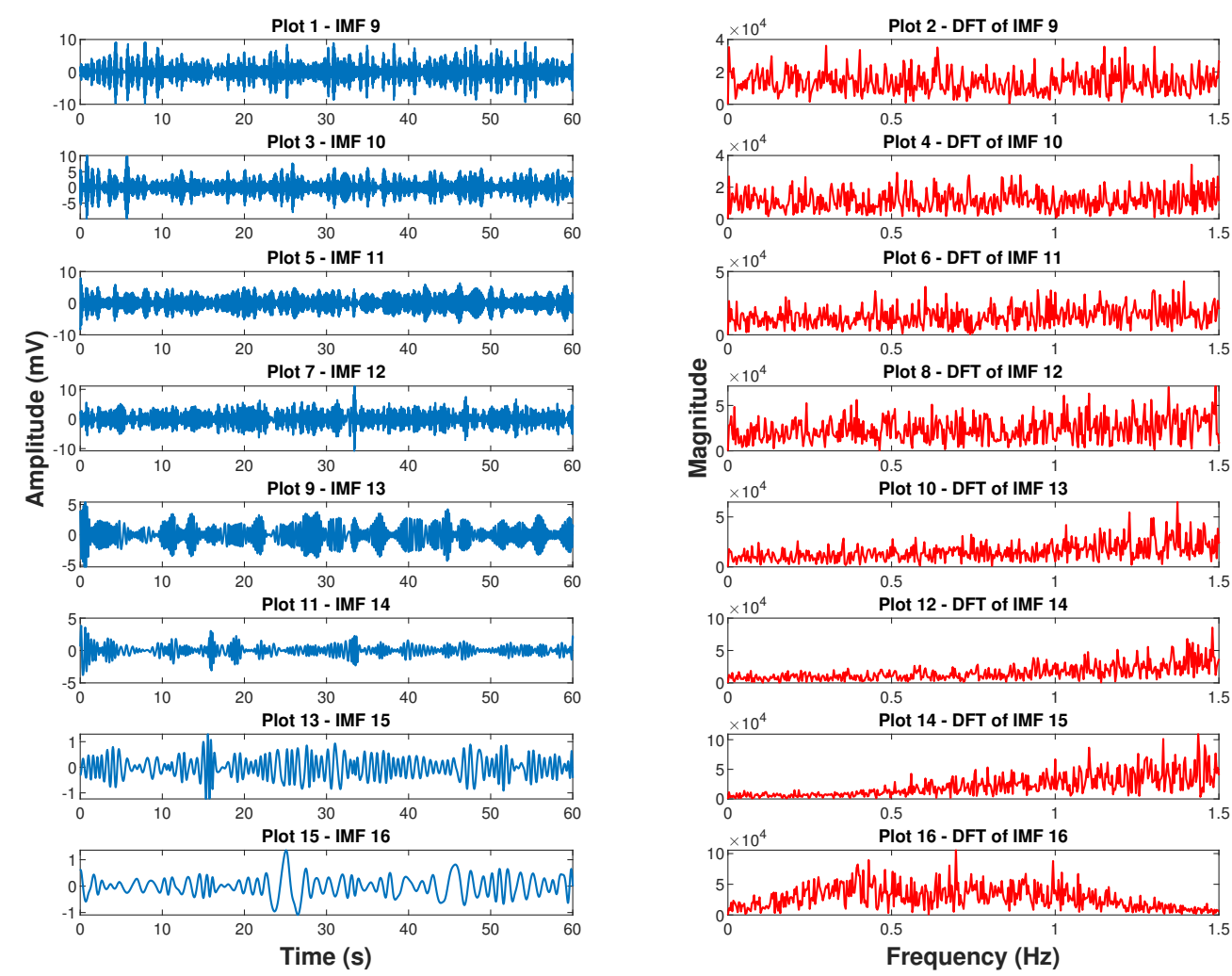

Figure 3. DFT analysis of the IMFs 9 to 16 of b001: The plots on the left column depict IMFs 9 to 16. The plots on the right column show the corresponding spectra (DFT points $=300,000$ ).
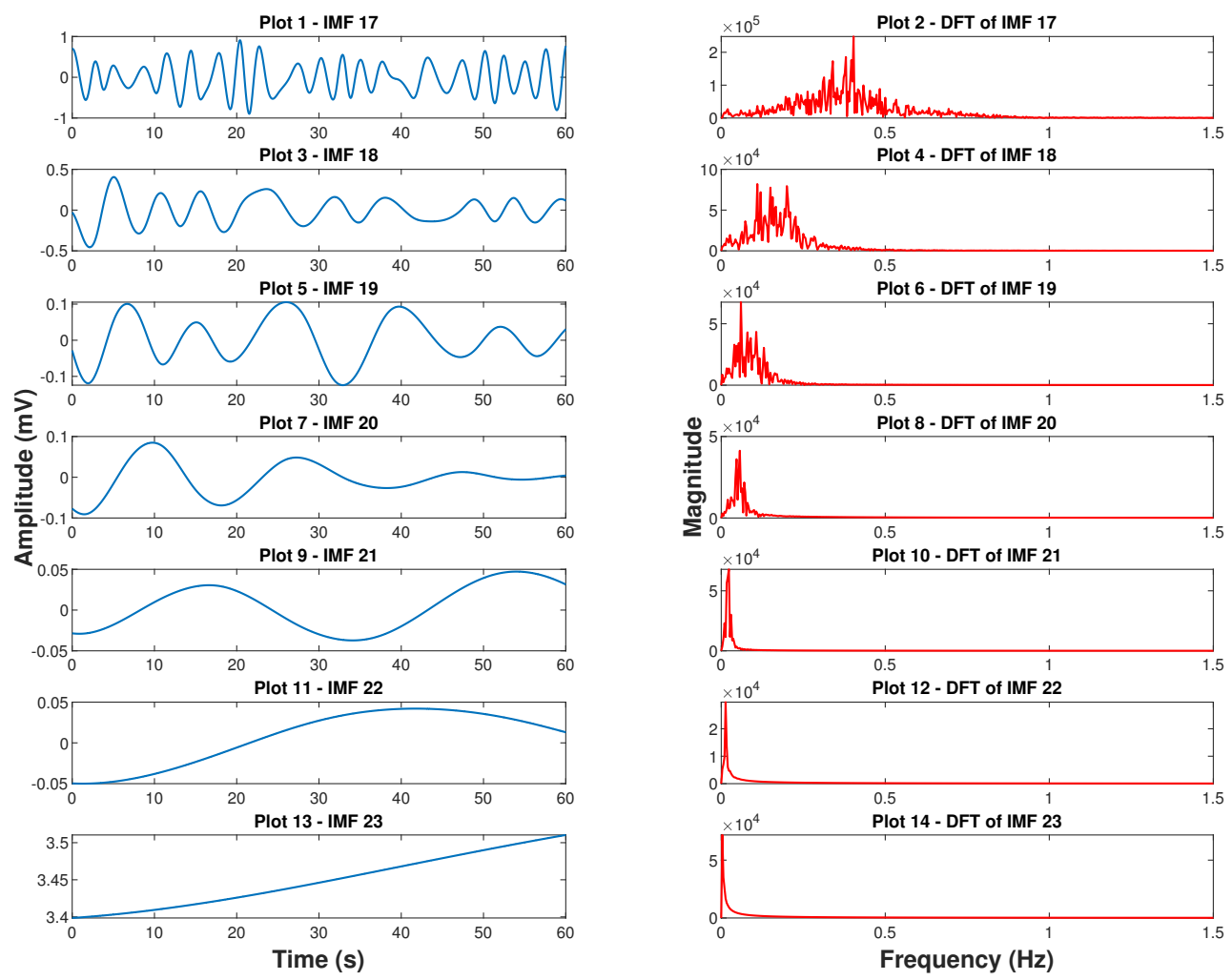

Figure 4. DFT analysis of the IMFs 17 to 23 of b001: The plots on the left column depict IMFs 17 to 23. The plots on the right column show the corresponding spectra (DFT points $=300,000$ ). 


\section{Time and Frequency Domain Analysis of Respiration Signals}

Thus far, this paper has discussed respiration signal extraction methods. As soon as the respiration signal is derived, it can be processed either in the time or in the frequency domain in order to obtain the BR estimates. According to the methodology followed in this work, the respiration signal is divided into one minute windows and each window is further analysed.

\subsection{Frequency Domain Analysis}

The main assumption of the frequency domain estimation of the BR from a respiratory signal is that the most dominant spectral peak of the respiratory signal corresponds to the BR. Under this assumption, the most commonly used method is the DFT. For the DFT analysis, the size of the transform, $N$, is defined as: $N=60 * f_{\text {resp }}$, where $f_{\text {resp }}$ is the sampling frequency of the respiration signal and 60 corresponds to the time duration of the one minute window in seconds. As previously discussed, the respiration signals are re-sampled at $8 \mathrm{~Hz}$, which results in an $N$ of 480 DFT points. To avoid repetition, additional information on the DFT analysis will be given when the respiration signal is not re-sampled and its frequency depends on the frequency of the SCG signal.

\subsection{Time Domain Analysis}

Time domain (TD) estimation analysis aims to detect individual breaths in the respiration signal by incorporating algorithms which detect peaks in the respiration signal. In [29], a method where the breaths in the respiration signal are identified using a three-point (3PT) scheme was proposed, and it is described in Algorithm 1.

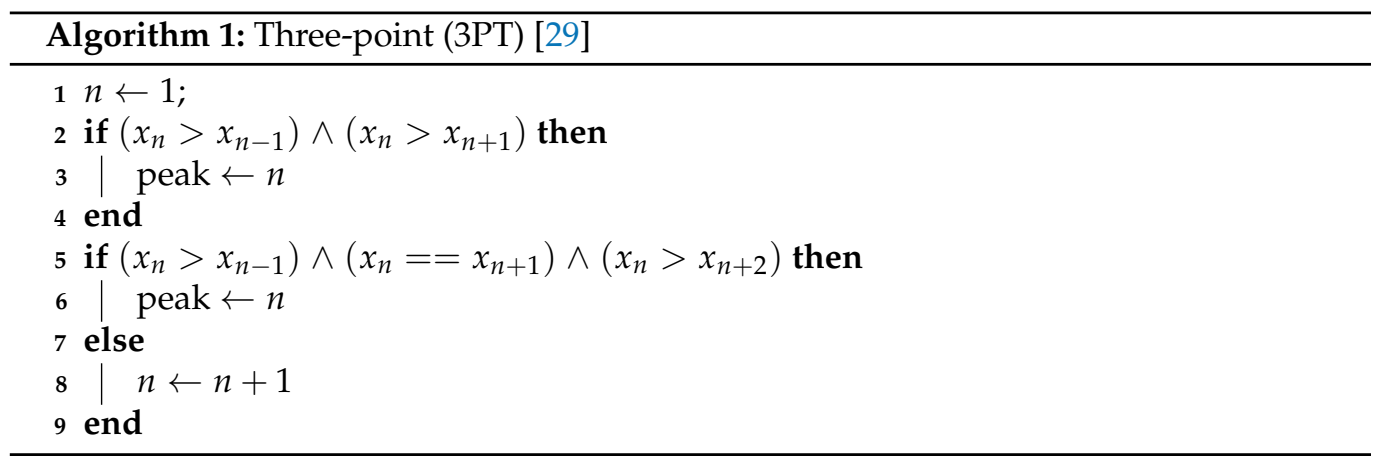

A more complicated method for the time domain BR estimation was suggested by [30]. The idea is to identify peaks and troughs in the respiration signal and then apply a set of rules in order to detect the final peaks which correspond to breaths. This algorithm will be referred from now on as peak-to-trough (P2T). A peak or trough is valid if it fulfills the following four rules:

a. A point is considered a peak if the gradient change is from positive to negative or a trough if the gradient change is from negative to positive,

b. A peak must be followed by a trough and vice versa,

c. The amplitude of a peak should be above the mean of the respiration signal and the amplitude of a trough should be below the mean,

d. The peak-to-peak or trough-to-trough interval should be greater than $0.5 \mathrm{~s}$,

As soon as the location of the breaths, $t_{1}, t_{2}, \ldots, t_{n}$ where $n$ is the total number of breaths, are identified using any of the two methods discussed above, the instantaneous $\mathrm{BR}$ (IBR) is computed as follows:

$$
\mathrm{IBR}_{i}=60 \times \frac{1}{t_{i+1}-t_{i}}
$$


where $i=1, \ldots, n-1$. Then, the IBR values are averaged over one minute windows in order to obtain the final BR estimates.

\section{Methods Evaluation}

The performance of the methods discussed in the previous sections was assessed by calculating the Mean Absolute Error (MAE) in bpm as follows:

$$
\mathrm{MAE}=\frac{1}{N} \sum_{i=1}^{N}\left|\mathrm{BR}_{\mathrm{ref}_{i}}-\mathrm{BR}_{\mathrm{est}_{i}}\right|,
$$

where $\mathrm{BR}_{\mathrm{ref}_{i}}$ is the reference $\mathrm{BR}, \mathrm{BR}_{\mathrm{est}}$ is the estimated $\mathrm{BR}$, and $N$ is the total number of one minute windows. Moreover, for each method, we provide a confidence interval (CI) which is given as $\pm 2 \sigma$, where $\sigma$ is the standard deviation of the MAEs for a specific method.

\section{Results}

\subsection{SCG-Derived BR: Standard Methods \\ 7.1.1. S1-S1 Interval Derived BR}

After successful extraction of the filtered S1-S1 respiration signal, the latter was further processed either in the frequency domain (DFT) or time domain (3PT and P2T) in order to obtain the BR estimates. Table 1 summarises the results obtained from the S1-S1 interval method for the CEBSDB recordings. It is observed that the method shows an adequate performance especially after the time domain analysis. The best performance is achieved by the 3PT time domain analysis, giving an average MAE of $3.2 \mathrm{bpm}$.

Figure 5 shows the frequency and time domain analysis of the S1-S1 interval signal obtained for subject b001. The reference BR of the first two respiratory one minute windows is $24 \mathrm{bpm}(0.4 \mathrm{~Hz})$. It can be seen in Plots 2 and 3 that the most dominant frequencies are far from the reference. This can be explained by the shape of the derived respiration signal (Plot 1). It is evident that the most dominant in amplitude oscillations in the first one minute window is 8 , which is equal to the most dominant frequency $(0.125 \mathrm{~Hz})$ in Plot 2. Similar behaviour is observed for the second window, where the number of the most dominant in amplitude oscillations is 5, which is the same as the most dominant frequency of $0.08 \mathrm{~Hz}$ in Plot 3. What is interesting about the frequency domain analysis is that the BR frequency $(0.4 \mathrm{~Hz})$ is still present. This combination of findings provides some support for the conceptual premise that the S1-S1 interval method does not capture the power of the respiratory-induced modulations of the SCG signal; consequently, the latter cannot be adequately reflected in the frequency spectrum of the respiration signal.

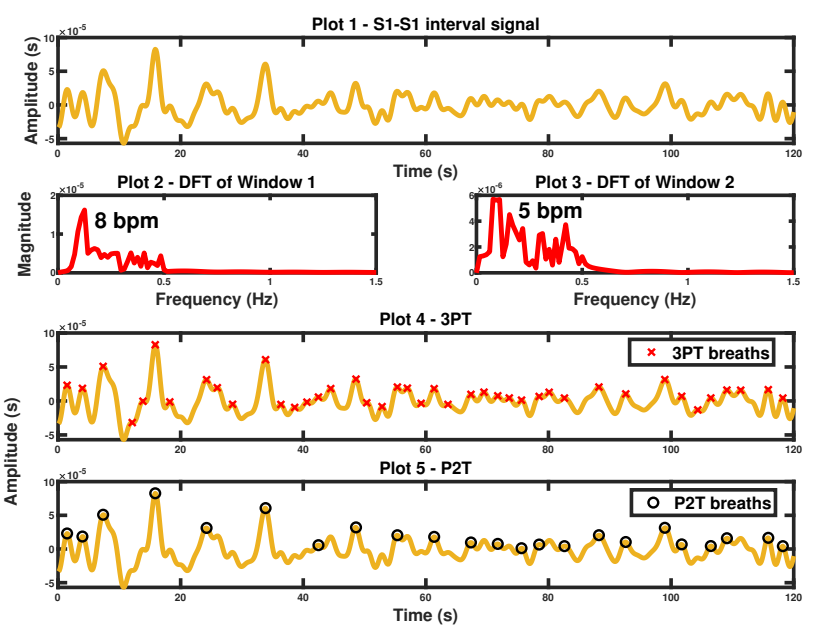

Figure 5. DFT and TD analysis of the S1-S1 interval signal on b001: Plot 1 shows the S1-S1 interval signal for subject b001. Plots 2 and 3 represent the DFT analysis. Plot 4 shows the breaths obtained from 3PT method as red crosses. Plot 5 shows the breaths obtained from P2T method as black circles. 
Table 1. S1-S1 interval performance.

\begin{tabular}{cccc}
\hline Record & MAE $_{\text {DFT }}(\mathbf{b p m})$ & MAE $_{3 \mathbf{P T}}(\mathbf{b p m})$ & MAE $_{\mathbf{P 2 T}}(\mathbf{b p m})$ \\
\hline b001 & 16.8 & 3 & 10.2 \\
b002 & 6.2 & 0.6 & 2.2 \\
b003 & 0.0 & 1.0 & 0.0 \\
b004 & 7.8 & 5.4 & 4.4 \\
b005 & 0.0 & 2.4 & 0.6 \\
b006 & 3.0 & 1.8 & 1.4 \\
b007 & 1.4 & 10.2 & 5.6 \\
b008 & 16.8 & 3 & 10.2 \\
b009 & 1.2 & 6.8 & 1.4 \\
b010 & 1.4 & 0.8 & 0.4 \\
b011 & 0.8 & 0.6 & 1.4 \\
b012 & 17 & 1.2 & 10.4 \\
b013 & 14.6 & 2.2 & 8.8 \\
b014 & 7.2 & 0.8 & 1.4 \\
b015 & 11.0 & 2.2 & 6.4 \\
b016 & 5.2 & 2.0 & 3.0 \\
b017 & 2.4 & 0.4 & 1.8 \\
b018 & 2.0 & 9.4 & 4.0 \\
b019 & 2.6 & 0.6 & 1.4 \\
b020 & 1.4 & 10.4 & 2.2 \\
\hline Average & 5.9 & 3.2 & 3.9 \\
\hline CI & \pm 12.1 & \pm 6.7 & \pm 7.0 \\
\hline
\end{tabular}

\subsubsection{S1 Intensity and S2 Intensity Derived BR}

As previously mentioned in Section 3.2, additional respiration-dependent features were explored in the study conducted by [14], which are based on the intensity of the S1 and S2 primary sounds of the SCG signal. Tables 2-4 illustrate the results obtained from the S1, S2 intensity, along with their ratio (S1/S2 intensity). The respiration signals obtained from the three intensity measures were further processed in the frequency domain (DFT) and the time domain (3PT and P2T) for the estimation of the BRs. As can be seen from these tables, the intensity methods outperform the S1-S1 interval method. In accordance with the present results, the study of [14] demonstrated the superiority of the intensity methods. The best performance is achieved by the frequency domain analysis of the S2 intensity respiration signal, giving an average MAE of $1.6 \mathrm{bpm}$.

Figure 6 demonstrates in Plots 1 to 3 the S1, S2, and S1/S2 intensity respiration signals obtained for subject b001, respectively. It is apparent that these signals capture more adequately the respiratory oscillations, compared to the S1 interval signal (Figure 5 Plot 1), meaning that the amplitude of the non-respiratory oscillations does not overlap the power of the respiratory ones. This is also reflected in the frequency spectrum of the three respiration signals, which is shown in Figure 6 Plots 4 and 5. The reference BR of the first two respiratory windows is $24 \mathrm{bpm}(0.4 \mathrm{~Hz})$. As can be seen, the most dominant frequencies of the S2 intensity spectrum are at $0.4 \mathrm{~Hz}$. The same applies for the S1/S2 intensity spectrum, a finding which is expected as this signal is affected by the $\mathrm{S} 2$ intensity, thus its performance is expected to be similar.

Regarding the time domain methods, it is observed that, for the intensity signals, the P2T analysis provides the best results, compared to the 3PT analysis. However, the best performance is still achieved by the DFT analysis of the S2 intensity signal. The observed increased MAE of the $3 \mathrm{PT}$ analysis can be attributed to the fact that the latter identifies as breaths all maxima present in the respiration signal, returning a higher BR. Furthermore, the more sophisticated analysis of P2T provides comparable to DFT analysis results because 
not all the maxima of the signal are detected as breaths due to the additional criteria of the P2T method.

Table 2. S1 Intensity performance.

\begin{tabular}{cccc}
\hline Record & MAE $_{\mathbf{D F T}}(\mathbf{b p m})$ & MAE $_{3 \mathbf{P T}}(\mathbf{b p m})$ & MAE $_{\mathbf{P 2 T}} \mathbf{( b p m )}$ \\
\hline b001 & 10.0 & 1.8 & 3.4 \\
b002 & 0.6 & 2.4 & 1.0 \\
b003 & 0.0 & 0.6 & 0.2 \\
b004 & 2.2 & 7.0 & 2.2 \\
b005 & 0.0 & 2.8 & 0.8 \\
b006 & 3.0 & 6.2 & 1.8 \\
b007 & 1.6 & 6.6 & 3.2 \\
b008 & 10 & 1.8 & 3.4 \\
b009 & 1.2 & 12.0 & 2.4 \\
b010 & 1.8 & 6.0 & 1.2 \\
b011 & 0.2 & 4.4 & 1.0 \\
b012 & 15.6 & 1.2 & 4.8 \\
b013 & 2.6 & 0.6 & 0.6 \\
b014 & 0.4 & 2.4 & 1.2 \\
b015 & 1.0 & 0.4 & 0.8 \\
b016 & 5.8 & 5.4 & 2.0 \\
b017 & 0.3 & 0.4 & 0.8 \\
b018 & 4.4 & 12.8 & 4.2 \\
b019 & 1.0 & 1.0 & 0.6 \\
b020 & 0.6 & 9.0 & 1.4 \\
\hline Average & 3.1 & 4.2 & $\mathbf{1 . 9}$ \\
\hline CI & \pm 8.4 & \pm 7.6 & \pm 2.6 \\
\hline
\end{tabular}

Table 3. S2 Intensity performance.

\begin{tabular}{cccc}
\hline Record & MAE $_{\text {DFT }}(\mathbf{b p m})$ & MAE $_{\text {3PT }}(\mathbf{b p m})$ & MAE $_{\text {P2T }}(\mathbf{b p m})$ \\
\hline b001 & 3.2 & 1.4 & 2.2 \\
b002 & 0.4 & 2.0 & 0.6 \\
b003 & 1.6 & 4.2 & 1.8 \\
b004 & 0.2 & 6.0 & 2.0 \\
b005 & 0.0 & 1.0 & 0.6 \\
b006 & 1.4 & 6.6 & 2.4 \\
b007 & 1.4 & 6.0 & 2.4 \\
b008 & 3.2 & 1.2 & 2.2 \\
b009 & 6.0 & 15.6 & 7.2 \\
b010 & 1.0 & 4.6 & 1.0 \\
b011 & 0.0 & 1.8 & 0.6 \\
b012 & 2.2 & 0.6 & 3.0 \\
b013 & 0.6 & 0.8 & 0.8 \\
b014 & 1.2 & 3.4 & 1.4 \\
b015 & 1.0 & 0.6 & 0.6 \\
b016 & 3.4 & 4.8 & 3.4 \\
b017 & 0.2 & 0.4 & 0.6 \\
b018 & 3.4 & 10.0 & 3.0 \\
b019 & 1.6 & 0.8 & 0.6 \\
b020 & 0.8 & 8.0 & 4.4 \\
\hline Average & $\mathbf{1 . 6}$ & $\mathbf{4 . 0}$ & $\mathbf{2 . 0}$ \\
\hline CI & \pm 3.1 & \pm 7.8 & \pm 3.3 \\
\hline
\end{tabular}


Table 4. S1/S2 Intensity performance.

\begin{tabular}{cccc}
\hline Record & MAE $_{\mathbf{D F T}}(\mathbf{b p m})$ & MAE $_{\text {3PT }}(\mathbf{b p m})$ & MAE $_{\mathbf{P 2 T}} \mathbf{( b p m )}$ \\
\hline b001 & 1.6 & 1.8 & 2.6 \\
b002 & 1.2 & 2.8 & 0.8 \\
b003 & 0.2 & 3.6 & 1.2 \\
b004 & 3.0 & 6.8 & 1.4 \\
b005 & 0.0 & 2.8 & 1.0 \\
b006 & 2.4 & 5.4 & 1.8 \\
b007 & 0.4 & 6.0 & 2.0 \\
b008 & 1.6 & 1.8 & 2.4 \\
b009 & 1.6 & 13.4 & 4.6 \\
b010 & 1.6 & 3.4 & 1.8 \\
b011 & 0.4 & 3.6 & 1.0 \\
b012 & 6.6 & 1.0 & 4.8 \\
b013 & 2.6 & 0.6 & 0.6 \\
b014 & 3.6 & 4.0 & 1.8 \\
b015 & 0.8 & 1.0 & 0.8 \\
b016 & 4.8 & 6.0 & 2.6 \\
b017 & 0.2 & 0.6 & 0.4 \\
b018 & 1.6 & 11.6 & 5.8 \\
b019 & 1.8 & 1.6 & 1.0 \\
b020 & 0.2 & 10.2 & 3.0 \\
\hline Average & $\mathbf{1 . 8}$ & 4.4 & $\mathbf{2 . 1}$ \\
\hline CI & \pm 3.4 & \pm 7.4 & \pm 3.0 \\
\hline
\end{tabular}
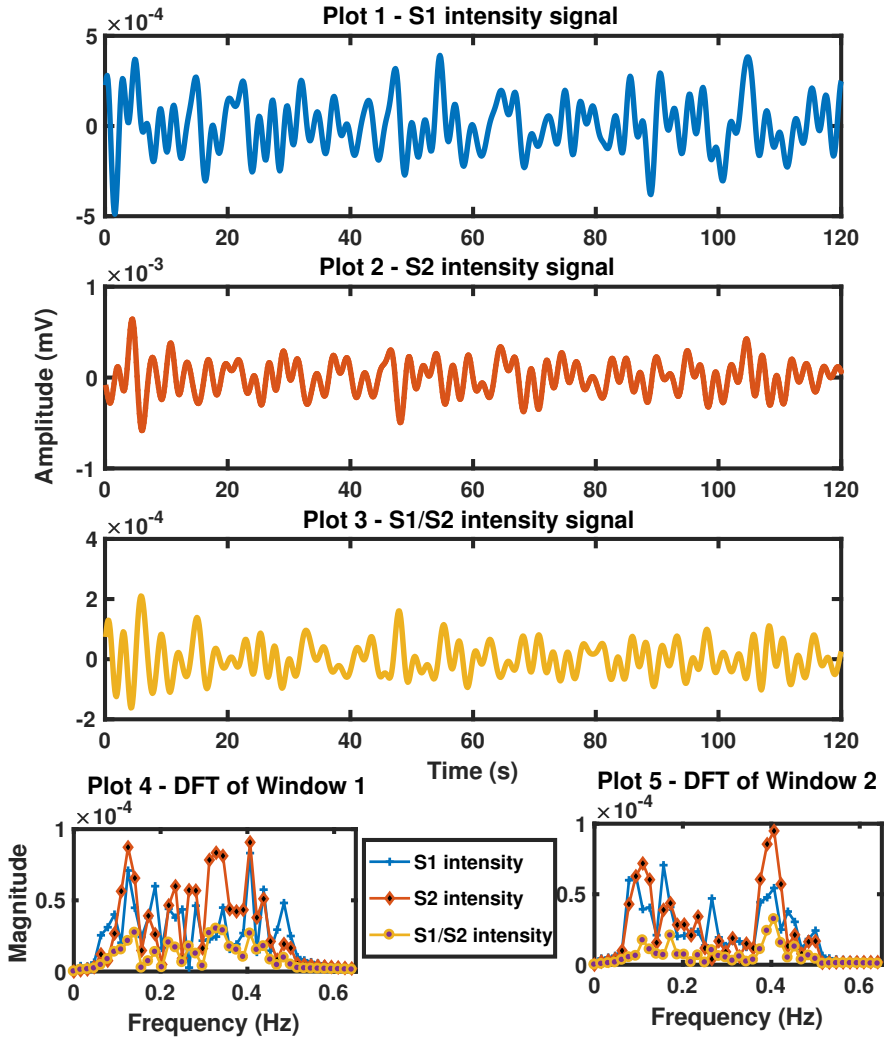

Figure 6. DFT analysis of the intensity methods on b001: Plots 1 and 2 show the S1 and S2 intensity signals, respectively. Plot 3 depicts the S1/S2 intensity signal. Plot 4 shows the DFT analysis of the three methods for the first respiratory window, where Plot 5 gives the corresponding information for the second respiratory window. 


\subsection{SCG-Derived BR: Proposed Advanced Methods}

\subsubsection{S1 Peak Amplitude Modulation Derived BR}

This section demonstrates the achieved accuracy performance of the AM method when analysed in both frequency and time domain. Table 5 summarises the results obtained for the CEBSDB data using the proposed S1 Peak Amplitude Modulation method. It is observed that the best performance is achieved by the DFT analysis of the AM S1 respiration signal, giving an average MAE of $3.2 \mathrm{bpm}$. It is interesting to note that these results are very close to the ones obtained from the $\mathrm{S} 1$ intensity signal. A possible explanation for this might be that both methods are taking advantage of the amplitude information of either the S1-peak or its RMS values over a pre-specified interval. However, the proposed method is computationally less intensive as it does not require further processing to calculate RMS values and at the same time achieves similar accuracy.

Table 5. S1 AM performance.

\begin{tabular}{cccc}
\hline Record & MAE $_{\mathbf{D F T}}(\mathbf{b p m})$ & $\mathbf{M A E}_{3 \mathbf{P T}}(\mathbf{b p m})$ & $\mathbf{M A E}_{\mathbf{P 2 T}}(\mathbf{b p m})$ \\
\hline b001 & 4.4 & 1.4 & 4.8 \\
b002 & 1.2 & 5.0 & 1.8 \\
b003 & 0.0 & 0.2 & 0.2 \\
b004 & 6.8 & 8.4 & 2.2 \\
b005 & 8.6 & 6.6 & 2.8 \\
b006 & 5.4 & 7.6 & 2.8 \\
b007 & 1.6 & 7.8 & 3.2 \\
b008 & 4.4 & 1.4 & 4.6 \\
b009 & 4.2 & 13.8 & 5.6 \\
b010 & 1.6 & 7.2 & 2.6 \\
b011 & 0.4 & 3.0 & 3.2 \\
b012 & 3.6 & 1.6 & 3.2 \\
b013 & 8.4 & 1.0 & 3.8 \\
b014 & 1.4 & 2.0 & 0.8 \\
b015 & 1.0 & 2.6 & 1.0 \\
b016 & 1.6 & 5.2 & 3.8 \\
b017 & 0.2 & 0.6 & 0.8 \\
b018 & 2.4 & 10.6 & 5.4 \\
b019 & 6.0 & 1.0 & 2.8 \\
b020 & 1.4 & 9.2 & 4.2 \\
\hline Average & 3.2 & 4.8 & 3.9 \\
\hline CI & \pm 5.4 & \pm 7.8 & \pm 3.1 \\
\hline
\end{tabular}

Figure 7 shows the DFT analysis of the first two respiratory windows of the AM S1 signal for subject b001. The reference BR of these windows is at $24 \mathrm{bpm}(0.4 \mathrm{~Hz})$. It can be seen from Plot 2 that the most dominant peak is at $0.1667 \mathrm{~Hz}(10 \mathrm{bpm})$, which is quite far from the reference. This can be possibly justified by the shape of the obtained respiration signal in the first $60 \mathrm{sec}$, where the slower oscillations seem to be more prominent in amplitude, compared to the fast ones which are related to respiration. 

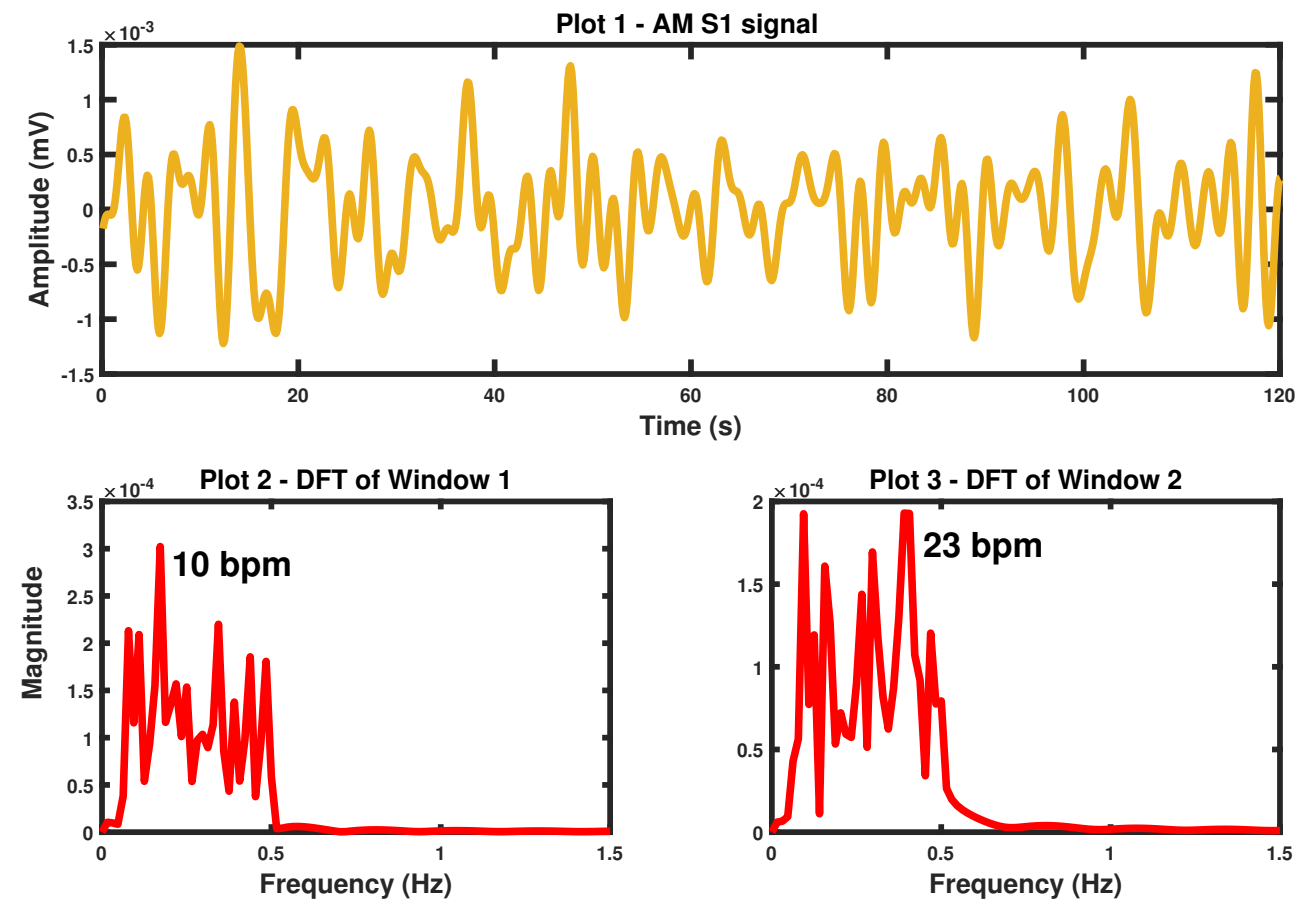

Figure 7. DFT analysis of the AM S1 signal on b001: Plot 1 shows the obtained AM S1 signal. Plots 2 and 3 depict the DFT analysis of the first two respiratory windows.

\subsubsection{Empirical Mode Decomposition Derived BR}

As previously mentioned, this paper examines for the first time the respiration signal extraction from an SCG using the EMD analysis. The results are summarised in Table 6 for both frequency and time domain analysis. The overall response of the EMDDR method on the SCG is very promising, as it outperforms all the state-of-the-art and the AM S1 method proposed in this work, achieving a low average MAE of $1.5 \mathrm{bpm}$ for the DFT analysis. This is an interesting finding which can be explained by the fact that this method does not depend on the S1-peaks, whose identification is not well defined, as there is a lack of studies which clarify their detection.

Figure 8 illustrates the DFT and time domain analysis of the EMDDR signal obtained for subject b001. The reference BR of the first two respiratory windows is $24 \mathrm{bpm}(0.4 \mathrm{~Hz})$. It is evident from Plots 1 and 2 that the most dominant spectral peak is located at $0.4 \mathrm{~Hz}$ (24 bpm), achieving a low error for these windows.

Overall, the present results on the EMDDR method using SCG data are encouraging and significant in at least two major respects: (1) the proposed EMDDR method does not involve the detection of the S1-peaks; consequently, the identification of the R-peaks is not needed, making the BR estimation less computationally expensive and less prone to R-peak detection artefacts, and (2) the EMDDR method does not require the re-sampling of the respiration signal as the latter is uniformly sampled at the SCG sampling frequency. Moreover, the results of this section indicate that further investigations can be done in order to improve the BR estimation from the SCG data. The next section, therefore, moves on to discussing the application of fusion techniques on the SCG signals. 
Table 6. EMDDR performance.

\begin{tabular}{cccc}
\hline Record & MAE $_{\text {DFT }}(\mathbf{b p m})$ & MAE $_{\text {3PT }} \mathbf{( b p m )}$ & MAE $_{\mathbf{P} \mathbf{~}} \mathbf{( b \mathbf { b } )}$ \\
\hline b001 & 0.6 & 2.6 & 2.6 \\
b002 & 1.2 & 1.6 & 1.6 \\
b003 & 0.2 & 7.6 & 6.2 \\
b004 & 1.6 & 2.6 & 2.4 \\
b005 & 0.6 & 2.0 & 2.0 \\
b006 & 2.2 & 21.4 & 21.4 \\
b007 & 2.4 & 9.0 & 9.0 \\
b008 & 0.6 & 2.6 & 2.6 \\
b009 & 0.8 & 1.0 & 0.8 \\
b010 & 1.6 & 1.8 & 1.4 \\
b011 & 2.6 & 3.0 & 3.0 \\
b012 & 3.4 & 3.4 & 3.4 \\
b013 & 1.4 & 3.8 & 3.8 \\
b014 & 0.2 & 2.8 & 2.8 \\
b015 & 1.6 & 2.0 & 2.0 \\
b016 & 4.4 & 4.0 & 3.8 \\
b017 & 0.0 & 1.0 & 1.0 \\
b018 & 3.6 & 4.0 & 4.0 \\
b019 & 0.2 & 4.2 & 4.2 \\
b020 & 0.8 & 5.0 & 4.6 \\
\hline Average & $\mathbf{1 . 5}$ & 4.3 & 4.1 \\
\hline CI & \pm 2.5 & \pm 9.0 & \pm 9.0 \\
\hline
\end{tabular}
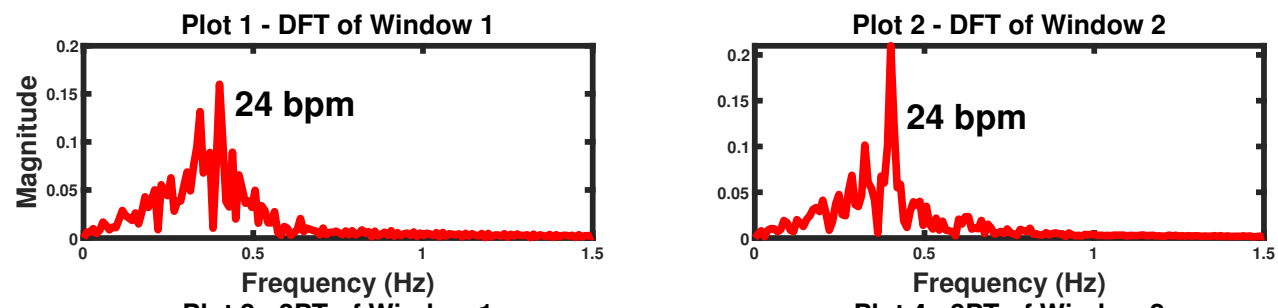

Plot 3 - 3PT of Window

Plot 4 - 3PT of Window 2

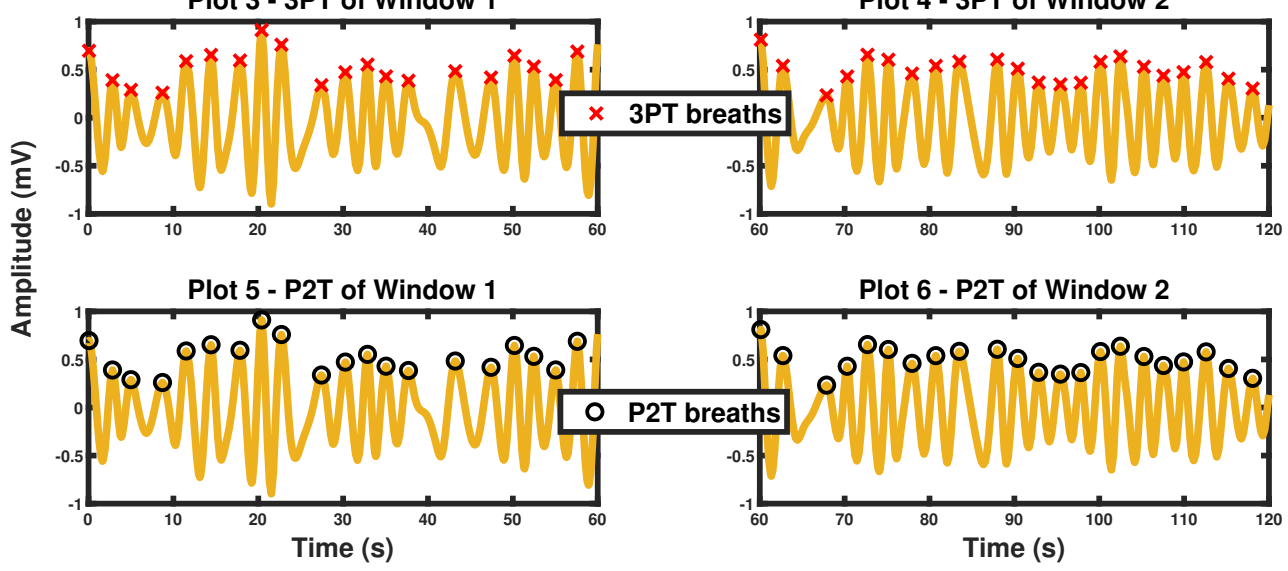

Figure 8. DFT and TD analysis of the EMDDR signal of b001: Plots 1 and 2 depict the DFT analysis of the first two respiratory windows (DFT points $=300,000$ ). Plots 3 and 4 show the identified breaths (red crosses) by the 3PT analysis. Plots 5 and 6 represent the identified breaths (black circles) by the P2T analysis.

\section{Proposed Method Fusion and Results}

It has been reported in the literature that BR estimation accuracy might be improved by using method fusion techniques. The main idea is to generate respiration signals 
or BR estimates from several methods and fuse them either in the time or frequency domain $[23,31,32]$. In the majority of studies, an all-pole AR model was used for the spectral analysis of the respiration signals and a pole magnitude criterion was developed for the method fusion $[23,33,34]$. The idea of this method is based on the fact that the spectral peaks of the signal are represented by the poles of the AR model [35]. Moreover, the frequencies of the spectral peaks are given by the phase angle of the corresponding poles of the model.

Regarding the method fusion for the BR estimation, respiratory signals from different methods were derived. Each respiratory signal was modelled using an all-pole AR model. A respiratory pole for each signal was selected based an a magnitude and phase angle criterion. First, the poles and phase angles are evaluated. The poles whose phase angle falls within reasonable respiration frequencies are kept and their magnitude is computed. The candidate respiratory poles are selected based on a magnitude criterion, and the final $\mathrm{BR}$ pole is set to be the pole with the minimum phase angle, which corresponds to the minimum frequency. Hence, at this stage, different BR poles are derived, one for each generated respiration signal. Finally, the fused respiratory pole is set to be the pole with the highest magnitude, thus the final selection is based on a pole magnitude criterion.

As was pointed out in the Introduction, the main limitation of method fusion techniques that use AR modelling is the model order selection. In [23], an all-pole AR model is designed for the spectral analysis of the respiration signals produced by different methods. After testing models with orders ranging from 6 to 20, Ref. [23] selected the model order to be 8 , as it gave the best results. Nonetheless, this method can be seen to be age and be subject specific and hence the estimation accuracy can be deteriorated when using a different dataset.

Therefore, a model order selection scheme is proposed in this work based on the PACF of the respiration signals. The PACF gives an image of the relationship between a current value of the signal with its values at prior time steps, and it is commonly used in AR model order selection [36]. The idea is to select as a model order the first lag where the PACF falls under 0.2 , thus using an $80 \%$ confidence interval. Algorithm 2 summarises the improved method fusion technique based on the pole magnitude and phase angle criterion of an AR model.

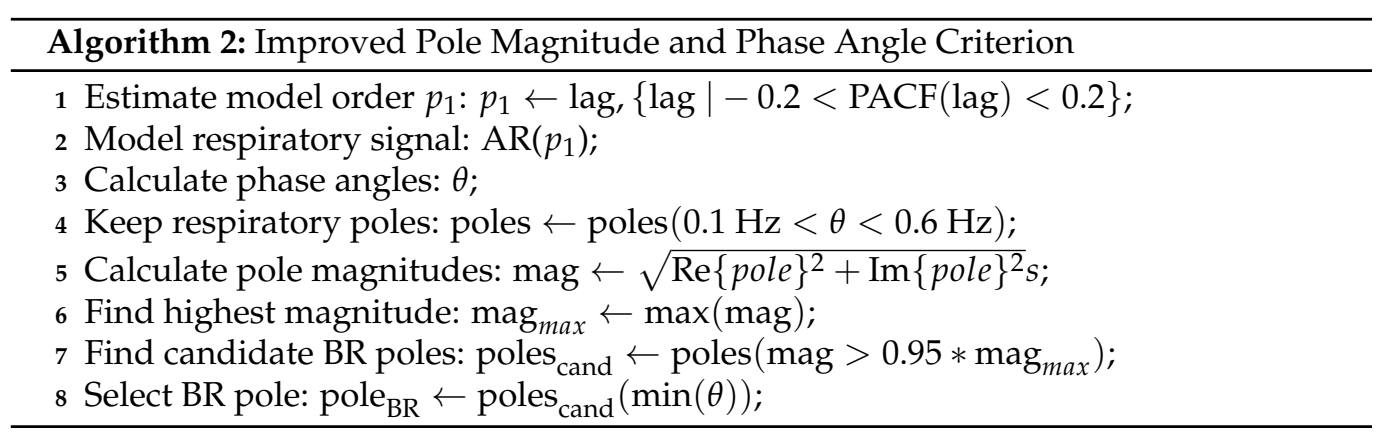

The results obtained from the AR-based method fusion, fusing respiratory signals over one minute window from the S1-S1 interval, S1, S2, and S1/S2 intensity, and the AM S1 methods are illustrated in Table 7. The statistical analysis reveals that the method fusion achieved a low MAE of $1.5 \mathrm{bpm}$, which outperforms the single intensity and AM S1 methods. Furthermore, during the experiments, it was observed that the total number of respiratory windows produced for the CEBSDB data are 100 . The method chose $40 \%$ of the windows to get the S2 intensity estimate, $25 \%$ to get the S1 intensity, $16 \%$ to get the S1/S2 intensity, $11 \%$ to get the AM S1 estimate, and $8 \%$ to get the S1-S1 interval estimate. This observation is also illustrated in Figure 9. This also accords with our earlier observations for the intensity and S1-S1 interval methods, which showed that intensity methods outperform the S1-peak time variation method, which is consistent with the study of [14]. Therefore, the method fusion correctly selects more BR estimates extracted by the intensity methods. 
Table 7. Method Fusion performance.

\begin{tabular}{cc}
\hline Record & MAE $_{\text {method fusion }}(\mathbf{b p m})$ \\
\hline b001 & 2.4 \\
b002 & 1.6 \\
b003 & 0.2 \\
b004 & 1.2 \\
b005 & 0.4 \\
b006 & 2.4 \\
b007 & 1.2 \\
b008 & 2.4 \\
b009 & 1.4 \\
b010 & 0.8 \\
b011 & 0.6 \\
b012 & 1.4 \\
b013 & 2.4 \\
b014 & 1.8 \\
b015 & 1.0 \\
b016 & 3.4 \\
b017 & 0.6 \\
b018 & 2.8 \\
b019 & 1.2 \\
b020 & 0.6 \\
\hline Average & $\mathbf{1 . 5}$ \\
\hline CI & \pm 1.8 \\
\hline
\end{tabular}

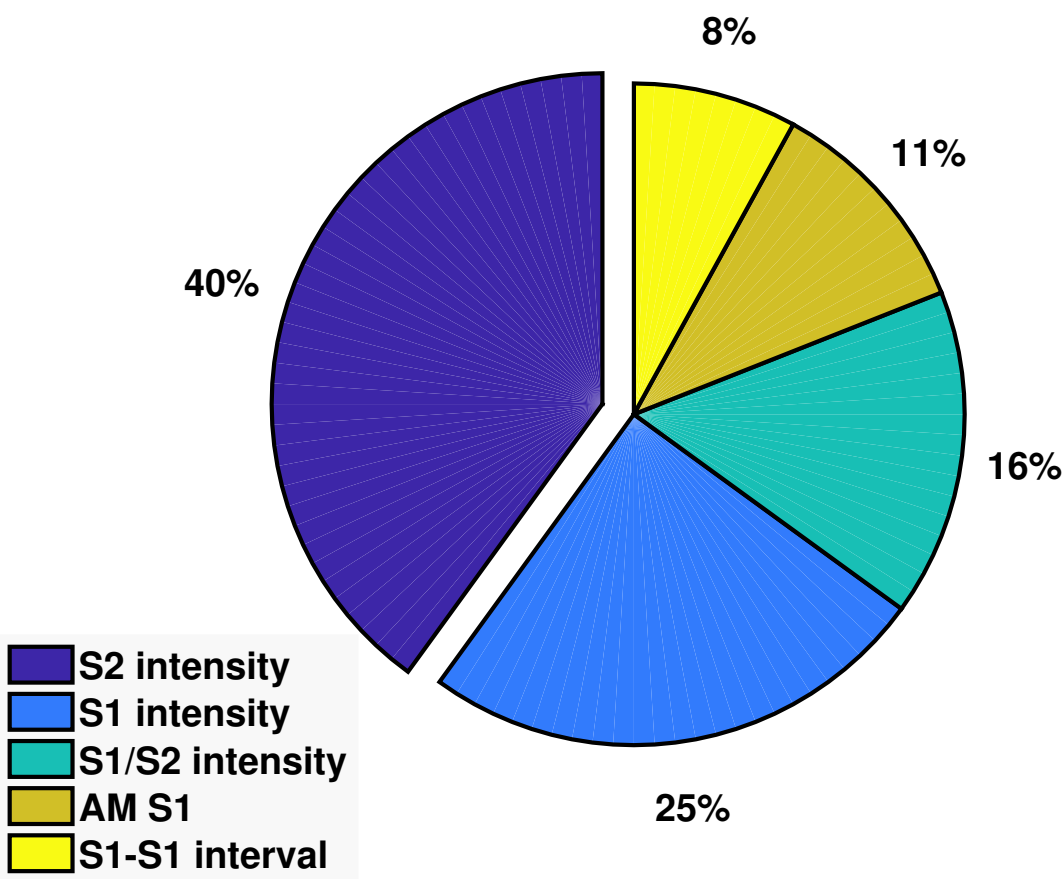

Figure 9. Distribution of respiratory windows based on method fusion.

\section{Further Analysis}

\subsection{Errors Associated with Gender and Differences in Lifestyle}

The database used in this study contains SCG recordings from 12 male and 8 female subjects. Moreover, 12 of the subjects were following a healthy lifestyle at the time of the recording, whereas the remaining 8 subjects were following a more sedentary lifestyle [24]. The results of comparison between male and female subjects for each developed in this 
work method are shown in Figure 10. We can observe that all the methods performed better for female compared to male subjects achieving lower MAEs. In addition, Figure 11 shows a MAE comparison for subjects with different lifestyles. It is evident that the majority of the developed methods show comparable performance. The latter indicates that these methods do not depend on the subject's state; therefore, they can be potentially applied to anyone regardless their clinical condition.

Another important finding that results from Figures 10 and 11 is the performance of the proposed fusion technique. As we can see, the fusion approach achieved similar MAEs for either male or female individuals. Similarly, the same performance was achieved for individuals with different lifestyles. This indicates that the fusion approach is not patient specific as the AR model order is based on the PACF of the respiration signal.

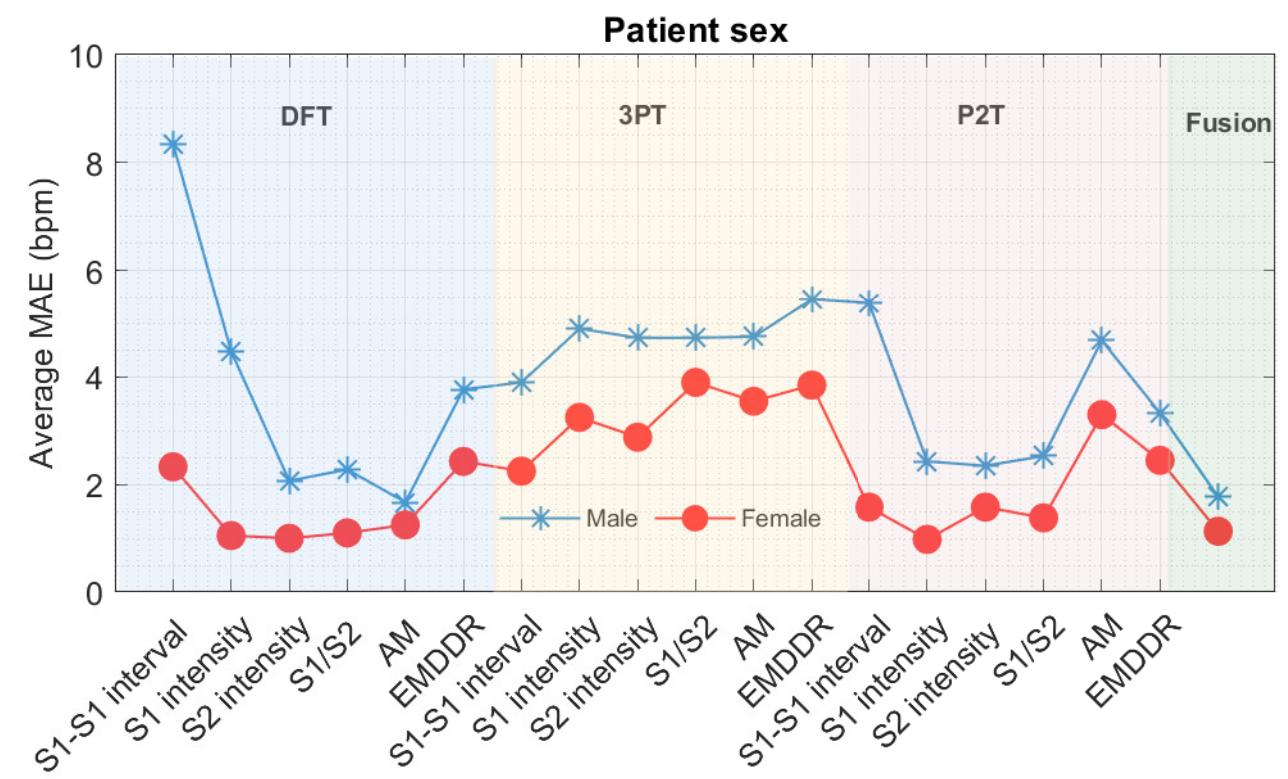

Figure 10. Comparison of MAE for male and female subjects.

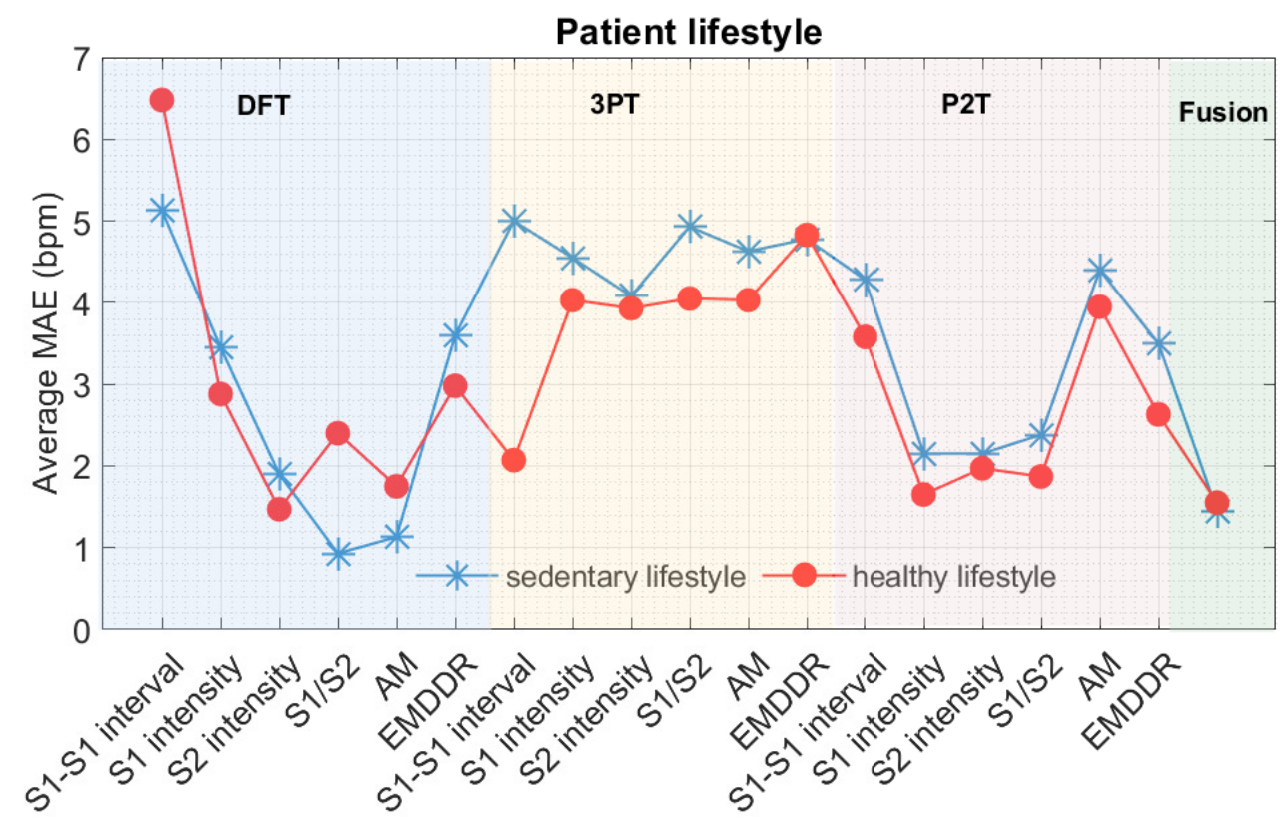

Figure 11. Comparison of MAE for subjects that followed a healthy or a sedentary lifestyle. 


\subsection{Errors Associated with Different Respiratory Rates}

Previous studies on SCG BR estimation have not dealt with how methods perform at lower and higher BRs. In this paper, we calculated the MAE for different ranges of BRs ( $\leq 12 \mathrm{bpm}$ ( $16 \%$ of respiratory windows), $12-16 \mathrm{bpm}$ (41\% of respiratory windows), $16-20 \mathrm{bpm}$ ( $22 \%$ of respiratory windows), and $\geq 20 \mathrm{bpm}$ ( $21 \%$ of respiratory windows)), as it would be interesting to see how methods behave when the subject increases or decreases its BR. The findings are summarised in Table 8.

Moreover, to gain a better understanding of the dynamics of the fusion approach, we calculated the percentage of respiratory windows for which the S1-S1 interval, S1 intensity, S2 intensity, S1/S2 or AM pole was the most dominant pole, for the different ranges of BRs, shown in Table 9.

Table 8. MAE in bpm and CI between different BRs estimated from the standard and advanced methods.

\begin{tabular}{ccccccc}
\hline $\begin{array}{c}\text { Range of } \\
\text { Reference BR }\end{array}$ & S1-S1 Interval & S1 Intensity & S2 Intensity & S1/S2 & EMDDR & AM \\
\hline & MAE (CI) & MAE (CI) & MAE (CI) & MAE (CI) & MAE (CI) & MAE (CI) \\
\hline$\leq 12 \mathrm{bpm}$ & $1.40( \pm 3.0)$ & $1.73( \pm 3.8)$ & $2.53( \pm 7.9)$ & $1.47( \pm 3.1)$ & $1.80( \pm 2.8)$ & $2.93( \pm 7.3)$ \\
$12-16 \mathrm{bpm}$ & $3.22( \pm 7.7)$ & $1.39( \pm 5.1)$ & $1.29( \pm 4.3)$ & $1.54( \pm 4.7)$ & $1.51( \pm 3.4)$ & $2.83( \pm 7.6)$ \\
$16-20 \mathrm{bpm}$ & $7.18( \pm 12.6)$ & $2.55( \pm 9.0)$ & $0.82( \pm 2.5)$ & $1.27( \pm 4.2)$ & $0.64( \pm 2.2)$ & $2.77( \pm 9.2)$ \\
$\geq 20 \mathrm{bpm}$ & $13.41( \pm 13.1)$ & $7.82( \pm 14.4)$ & $2.59( \pm 9.5)$ & $3.41( \pm 9.8)$ & $1.73( \pm 6.2)$ & $4.91( \pm 12.2)$ \\
\hline
\end{tabular}

Table 9. Percentage of respiratory poles identified by the proposed method fusion technique from either one of the respiratory waveforms.

\begin{tabular}{cccccc}
\hline $\begin{array}{c}\text { Range of } \\
\text { Reference BR }\end{array}$ & S1-S1 Interval (\%) & S1 Intensity (\%) & S2 Intensity (\%) & S1/S2 (\%) & AM (\%) \\
\hline <12 bpm & 18.75 & 37.50 & 18.75 & 18.75 & 6.25 \\
$12-16$ bpm & 12.20 & 19.51 & 34.15 & 14.63 & 19.51 \\
$16-20 \mathrm{bpm}$ & - & 36.36 & 50.00 & 9.09 & 4.55 \\
$\geq 20 \mathrm{bpm}$ & - & 14.29 & 52.83 & 23.81 & 9.52 \\
\hline
\end{tabular}

\section{Discussion}

This paper has provided a deeper insight into SCG BR estimation by proposing and demonstrating three novel methods for extracting the BR from SCG recordings. The first proposed method is shown to be dependent on the accurate identification of S1-peaks which depend on the detection of the R-peaks in the ECG signal. The results from our second proposed EMDDR method demonstrated a novel, clinically useful approach to monitor the BR in a continuous, non-invasive, and easy to use manner as it does not require any preprocessing of the SCG recordings. Furthermore, this proposed method achieved promising low MAEs between different ranges of breathing rates (Table 8 ). This indicates that EMDDR can be applied to anyone, regardless of how slow or fast they are breathing. Moreover, from an implementation point of view, EMD is straight-forward. The latter paves the way to design sensor/devices which measure both SCG and ECG in order to obtain two respiration signals using the EMDDR method, which can be later fused and provide a more robust BR estimation approach. Finally, the results obtained from our proposed method fusion technique showed that intensity methods are a more reliable source of respiratory information than time-based methods, which is also consistent with the study of [14]. This is also reflected in our findings in Table 9, where it is evident that, for different ranges of breathing rates in most cases, the most dominant pole results from an intensity-based method.

This study also showed that the majority of the analysed methods performed better for female compared to male subjects. As this has previously not been reported in any 
SCG-respiration related work, we speculate that the difference in anatomy (and, as a result, accelerometer placement) between male and female subjects might have an effect in the respiratory-related modulations in the SCG signal.

Finally, the current investigation has only examined the SCG BR estimation on healthy adults whose ages range from 19 to 30 years old. Further work will consider the application of the proposed methods to clinically deteriorated patients with more extreme BR. Additionally, while the proposed EMD method proved to be powerful in extracting the respiratory signal from SCG recordings, it is known that EMD is unstable to perturbations and susceptible to mode splitting and mode mixing issues. Therefore, our subsequent work will exploit the use, analyses, and enhancement of alternative EMD methods such as the Ensemble Empirical Mode Decomposition (EEMD) method [37] and noise-assisted EMD-based methods $[38,39]$ to give a few examples.

Author Contributions: Conceptualization, C.K. and R.H.; methodology, C.K. and R.H.; software, C.K.; validation, C.K.; formal analysis, C.K.; investigation, C.K. and R.H.; resources, C.K. and R.H.; data curation, C.K.; writing-original draft preparation, C.K.; writing-review and editing, R.H.; visualization, C.K.; supervision, R.H.; project administration, R.H.; funding acquisition, R.H. Both authors have read and agreed to the published version of the manuscript.

Funding: This research was funded by Isansys Lifecare Ltd. Grant No. NA.

Institutional Review Board Statement: Not applicable.

Informed Consent Statement: Not applicable.

Data Availability Statement: Not applicable.

Conflicts of Interest: The authors declare no conflict of interest.

\section{References}

1. Kelly, C. Respiratory Rate 1: Why measurement and recording are crucial. Nursing Times, 2 April 2018; pp. 23-24.

2. Cretikos, M.A.; Bellomo, R.; Hillman, K.; Chen, J.; Finfer, S.; Flabouris, A. Respiratory Rate: The neglected vital sign. Med. J. Aust. 2008, 188, 657-659. [CrossRef] [PubMed]

3. Fieselmann, J.F.; Hendryx, M.S.; Helms, C.M.; Wakefield, D.S. Respiratory Rate Predicts Cardiopulmonary Arrest for Internal Medicine Inpatients. J. Gen. Intern. Med. 1993, 8, 354-360. [CrossRef]

4. Goldhill, D.R.; McNarry, A.F.; Mandersloot, G.; McGinley, A. A Physiologically-based Early Warning Score for Ward Patients: The Association Between Score and Outcome. Anaesthesia 2005, 60, 547-553. [CrossRef] [PubMed]

5. Elliott, M. Why is Respiratory Rate the Neglected Vital Sign? A Narrative Review. Int. Arch. Nurs. Health Care 2016, 2, 50. [CrossRef]

6. Mlgaard, R.R.; Larsen, P.; Håkonsen, S.J. Effectiveness of Respiratory Rates in Determining Clinical Deterioration: A Systematic Review Protocol. JBI Database Syst. Rev. Implement. Rep. 2016, 14, 19-27. [CrossRef] [PubMed]

7. Charlton, P.H. Continuous Respiratory Rate Monitoring to Detect Clinical Deteriorations Using Wearable Sensors. Ph.D. Thesis, King's College London, London, UK, 2017.

8. Nayan, A.; Risman, N.; Jaafar, R. Breathing Rate Estimation from a Single-lead Electrocardiogram Acquisition System. Int. J. Appl. Eng. Res. 2015, 10, 38154-38158.

9. Charlton, P.H.; Bonnici, T.; Tarassenko, L.; Clifton, D.A.; Beale, R.; Watkinson, P.J. An assessment of algorithms to estimate respiratory rate from the Electrocardiogram and Photoplethysmogram. Physiol. Meas. 2016, 37, 610. [CrossRef]

10. Zakeri, V.; Akhbardeh, A.; Alamdari, N.; Fazel-Rezai, R.; Paukkunen, M.; Tavakolian, K. Analyzing Seismocardiogram cycles to identify the respiratory phases. IEEE Trans. Biomed. Eng. 2016, 64, 1786-1792. [CrossRef]

11. Haescher, M.; Matthies, D.J.C.; Trimpop, J.; Urban, B. A study on measuring heart-and respiration-rate via wrist-worn accelerometer-based seismocardiography (SCG) in comparison to commonly applied technologies. In Proceedings of the 2nd International Workshop on Sensor-based Activity Recognition and Interaction, Rostock, Germany, 25-26 June; Bodo Urban, Thomas Kirste, Association for Computing Machinery: New York, NY, USA, 2015; p. 2.

12. Tadi, M.J.; Koivisto, T.; Pänkäälä, M.; Paasio, A. Accelerometer-based method for extracting respiratory and cardiac gating information for dual gating during nuclear medicine imaging. J. Biomed. Imaging. 2014. [CrossRef]

13. Pandia, K.; Inan, O.T.; Kovacs, G.T.A. A frequency domain analysis of respiratory variations in the Seismocardiogram signal. In Proceedings of the 35th Annual International Conference of the IEEE Engineering in Medicine and Biology Society (EMBC), Osaka, Japan, 2-7 July 2013; pp. 6881-6884.

14. Pandia, K.; Inan, O.T.; Kovacs, G.T.A.; Giovangrandi, L. Extracting respiratory information from Seismocardiogram signals acquired on the chest using a miniature accelerometer. Physiol. Meas. 2012, 33, 1643. [CrossRef] 
15. Reinvuo, T.; Hannula, M.; Sorvoja, H.; Alasaarela, E.; Myllyla, R. Measurement of respiratory rate with high-resolution accelerometer and EMFit pressure sensor. In Proceedings of the 2006 IEEE Sensors Applications Symposium, Houston, TX, USA, 7-9 February 2006; pp. 192-195.

16. Inan, O.T.; Migeotte, P.F.; Park, K.S.; Etemadi, M.; Tavakolian, K.; Casanella, R.; Zanetti, J.; Tank, J.; Funtova, I.; Prisk, G.K.; et al. Ballistocardiography and Seismocardiography: A review of recent advances. IEEE J. Biomed. Health Inform. 2014, 19, $1414-1427$. [CrossRef]

17. Zanetti, J.M.; Salerno, D.M. Seismocardiography: A technique for recording precordial acceleration. In Proceedings of the Fourth Annual IEEE Symposium on Computer-Based Medical Systems, Baltimore, MD, USA, 12-14 May 1991; pp. 4-9.

18. Castiglioni, P.; Faini, A.; Parati, G.; Di Rienzo, M. Wearable Seismocardiography. In Proceedings of the 29th Annual International Conference of the IEEE Engineering in Medicine and Biology Society, Lyon, France, 23-26 August 2007; pp. $3954-3957$.

19. Karagiannis, A.; Constantinou, P. Noise components identification in biomedical signals based on Empirical Mode Decomposition. In Proceedings of the 9th International Conference on Information Technology and Applications in Biomedicine, Larnaca, Cyprus, 5-7 November 2009; pp. 1-4.

20. Civera, M.; Filosi, C.M.; Pugno, N.M.; Silvestrini, M.; Surace, C.; Worden, K. Assessment of vocal cord nodules: A case study in speech processing by using Hilbert-Huang Transform. J. Phys. Conf. Ser. 2017, 842, 012025. [CrossRef]

21. Civera, M.; Filosi, C.M.; Pugno, N.M.; Silvestrini, M.; Surace, C.; Worden, K. Using Wavelet Level Variance and the Discrete Wavelet Transform to Monitor Postoperative Healing of Vocal Cords. In Proceedings of the 9th European Workshop on Structural Health Monitoring (EWSHM 2018), Manchester, UK, 10-13 July 2018.

22. Rizi, F.Y. A Review of Notable Studies on Using Empirical Mode Decomposition for Biomedical Signal and Image Processing. Signal Process. Renew. Energy 2019, 3, 89-113.

23. Orphanidou, C.; Fleming, S.; Shah, S.A.; Tarassenko, L. Data fusion for estimating respiratory rate from a single-lead ECG. Biomed. Signal Process. Control. 2013, 8, 98-105. [CrossRef]

24. García-González, M.A.; Argelagós-Palau, A.; Fernández-Chimeno. M.; Ramos-Castro, J. A comparison of heartbeat detectors for the Seismocardiogram. In Proceedings of the Computing in Cardiology, Zaragosa, Spain, 22-25 September 2013; Alan Murray, IEEE: Piscataway, NJ, USA, 2013; pp. 461-464.

25. Goldberger, A.; Amaral, L.; Glass, L.; Hausdorff, J.; Ivanov, P.C.; Mark, R.; Mietus, J.E.; Moody, G.B.; Peng, C.K.; Stanley, H.E. PhysioBank, PhysioToolkit, and PhysioNet: Components of a new research resource for complex physiologic signals. Circulation [Online] 2000, 101, e215-e220. [CrossRef] [PubMed]

26. Kozia, C.; Herzallah, R.; Lowe, D. Adaptive R-peak Detection Using Empirical Mode Decomposition. In Proceedings of the International Conference on Time Series and Forecasting, ITISE 2018, Granada, Spain, 19-21 September 2018.

27. Kozia, C.; Herzallah, R.; Lowe, D. ECG-Derived Respiration Using a Real-Time QRS Detector Based on Empirical Mode Decomposition. In Proceedings of the 12th International Conference on Signal Processing and Communication Systems (ICSPCS), Cairns, Australia, 17-19 December 2018; pp. 1-8.

28. Kozia, C.; Herzallah, R.; Lowe, D. ICA-Derived Respiration Using an Adaptive R-Peak Detector. In Proceedings of the International Conference on Time Series and Forecasting, ITISE 2018, Granada, Spain, 19-21 September 2018; pp. 363-377.

29. Shah, S.A. Vital Sign Monitoring and Data Fusion for Paediatric Triage. Ph.D. Thesis, Oxford University, Oxford, UK, 2012.

30. Fleming, S. Measurement and Fusion of Non-Invasive Vital Signs for Routine Triage of Acute Paediatric Illness. Ph.D. Thesis, Oxford University, Oxford, UK, 2010.

31. Orphanidou, C. Derivation of respiration rate from ambulatory ECG and PPG using Ensemble Empirical Mode Decomposition: Comparison and Fusion. Comput. Biol. Med. 2017, 81, 45-54. [CrossRef] [PubMed]

32. Sobron, A.; Romero, I.; Lopetegi, T. Evaluation of methods for estimation of respiratory frequency from the ECG. In Proceedings of the Computing in Cardiology CinC 2010, Belfast, UK, 26-29 September 2010; pp. 513-516.

33. Nemati, S.; Malhotra, A.; Clifford, G.D. Data fusion for improved respiration rate estimation. EURASIP J. Adv. Signal Process. 2010. [CrossRef] [PubMed]

34. Fleming, S.G.; Tarassenko, L. A comparison of signal processing techniques for the extraction of breathing rate from the photoplethysmogram. Int. J. Biol. Med. Sci. 2007, 2, 232-236.

35. Proakis, J.G.; Manolakis, D.G. Digital Signal Processing: Principles, Algorithms, and Applications, 3rd ed.; Prentice-Hall, Inc.: Upper Saddle River, NJ, USA, 1996; p. 855.

36. Burke, O. Statistical Methods. Autocorrelation, Decomposition and Smoothing; Michaelmas Term. Department of Statistics: Oxford, UK, 2011.

37. Wu, Z.; Huang, N.E. Ensemble empirical mode decomposition: A noise-assisted data analysis method. Adv. Adapt. Data Anal. 2009, 1, 1-41. [CrossRef]

38. Yeh, J.R.; Shieh, J.S.; Huang, N.E. Complementary ensemble empirical mode decomposition: A novel noise enhanced data analysis method. Adv. Adapt. Data Anal. 2010, 2, 135-156. [CrossRef]

39. Zheng, J.; Cheng, J.; Yang, Y. Partly ensemble empirical mode decomposition: An improved noise-assisted method for eliminating mode mixing. Signal Process. 2014, 96, 362-374. [CrossRef] 